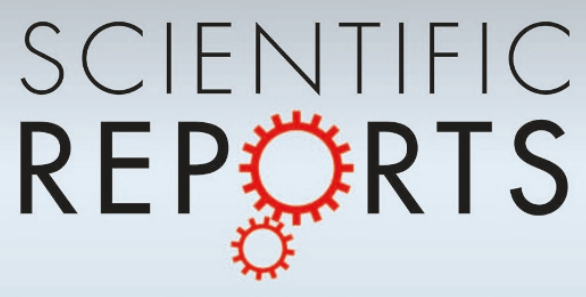

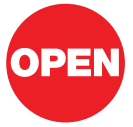

SUBJECT AREAS: COMPLEX NETWORKS

STATISTICAL PHYSICS

NONLINEAR PHENOMENA

INFORMATION THEORY AND COMPUTATION

Received

12 February 2013

Accepted

16 April 2013

Published

10 May 2013

Correspondence and requests for materials should be addressed to T.C. (its_tanmoy@cse. iitkgp.ernet.in)

\section{Constant Communities in Complex Networks}

\author{
Tanmoy Chakraborty', Sriram Srinivasan ${ }^{2}$, Niloy Ganguly', Sanjukta Bhowmick ${ }^{2}$ \& Animesh Mukherjee'
}

'Dept. of Computer Science \& Engg., Indian Institute of Technology, Kharagpur, India-721302, ${ }^{2}$ Dept. of Computer Science,
University of Nebraska, Omaha, Nebraska 68182.

Identifying community structure is a fundamental problem in network analysis. Most community detection algorithms are based on optimizing a combinatorial parameter, for example modularity. This optimization is generally NP-hard, thus merely changing the vertex order can alter their assignments to the community. However, there has been less study on how vertex ordering influences the results of the community detection algorithms. Here we identify and study the properties of invariant groups of vertices (constant communities) whose assignment to communities are, quite remarkably, not affected by vertex ordering. The percentage of constant communities can vary across different applications and based on empirical results we propose metrics to evaluate these communities. Using constant communities as a pre-processing step, one can significantly reduce the variation of the results. Finally, we present a case study on phoneme network and illustrate that constant communities, quite strikingly, form the core functional units of the larger communities.

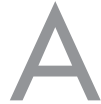

fundamental problem in understanding the behavior of complex networks is the ability to correctly detect communities. Communities are groups of entities (represented as vertices) that are more connected to each other as opposed to other entities in the system. Mathematically, this question can be translated to a combinatorial optimization problem with the goal of optimizing a given metric of interrelation, such as modularity or conductance. The goodness of community detection algorithms (see ${ }^{1,2}$ for a review) is often objectively measured according to how well they achieve the optimization.

However, these algorithms can be applied to any network, regardless of whether it possesses a community structure or not. Furthermore when the optimization problem is NP-hard, as in the case of modularity ${ }^{3}$, the order in which vertices are processed as well as the heuristics can change the results. These inherent fluctuations of the results associated with modularity have long been a source of concern among researchers. Indeed the goodness of modularity as an indicator of community structure has also been questioned, and there exist examples ${ }^{5}$ which demonstrate that high modularity does not always indicate the correct community structure. Other orthogonal graph-theoretic metrics (e.g., conductance ${ }^{6}$ which is also NP-complete ${ }^{4}$ ) have been proposed in the past literatures to judge the goodness of a community detection algorithm.

Research in addressing the fluctuations in the results due to modularity maximization heuristics include identifying stability among communities from the consensus networks built from the successive iterations of a non-deterministic community detection algorithm (such as by Seifi et al. ${ }^{7}$ ). Lancichinetti et al. ${ }^{8}$ proposed consensus clustering by reweighting the edges based on how many times the pair of vertices were allocated to the same community, for different identification methods. Delvenne et al..$^{9}$ introduced the notion of the stability of a partition, a measure of its quality as a community structure based on the clustered autocovariance of a dynamic Markov process taking place on the network. Delmotte et al. ${ }^{31}$ proposed a methodology based on multi-scale graph partitioning that can uncover partitions and levels of organization of proteins that span the whole range of scales, revealing biological features occurring at different levels of organization and tracking their effect across scales. They also proposed a measure of robustness to quantify the relevance of the partitions. Lai et al. ${ }^{10}$ proposed a random walk based approach to enhance the modularity of a community detection algorithm. Ovelgonne et al. ${ }^{11}$ pointed out an ensemble learning strategy for graph clustering. Gfeller et al. ${ }^{12}$ investigated the instabilities in the community structure of complex networks. Finally, several pre-processing techniques ${ }^{13,14}$ have been developed to improve the quality of the solution. These methods form an initial estimate of the community allocation over a small percentage of the vertices and then refine this estimate over successive steps.

All these methods focus on compiling the differences in the results to arrive at an acceptable solution, and despite these advances a crucial question about the variance of results remains unanswered - what do the 


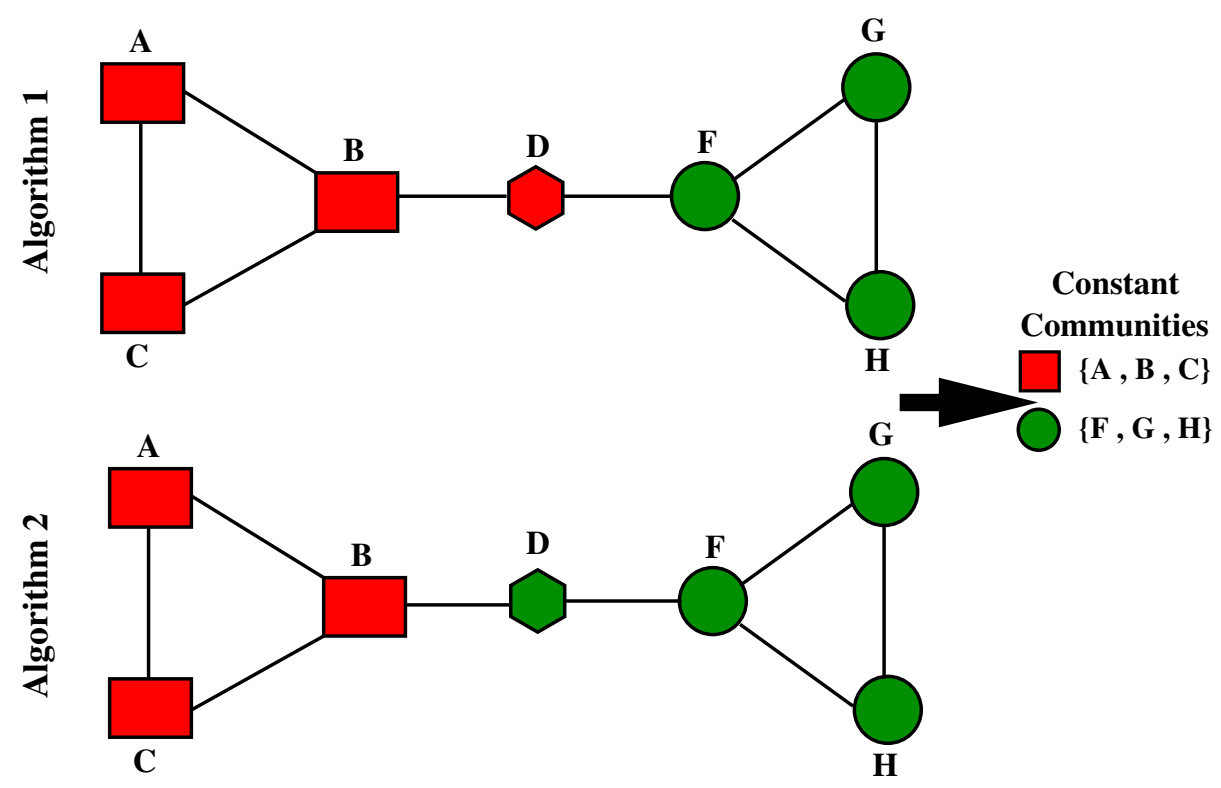

Figure $1 \mid$ Schematic illustration of the formation of constant communities. Two colors (red and green) indicate two communities of the network formed in each iteration. Combined results of two algorithms produce two constant communities (rectangular and circular vertices). Remaining one vertex (hexagonal shaped) is not included since it switches its community between the two algorithms.

invariance of the results tell us about the network structure? In this paper, we focus on the invariance in community detection as obtained by modularity maximization. Our results, on a set of scale-free networks, show that while the vertex orderings produce very different sets of communities, some groups of vertices are always allocated to the same community for all different orderings. We define the group of vertices that remain invariant as constant communities and the vertices that are part of the constant communities as constant vertices. Figure 1 shows a schematic diagram of constant communities. Note that not all vertices in the network belong to constant communities. This is a key difference of constant communities with the consensus methods ${ }^{8}$ described earlier. Consensus methods attempt to find the best (most stable or most similar) community among all available results and thus include all the vertices. Constant communities, on the other hand, focus on finding subgraphs where the cohesive groups can be unambiguously identified. As discussed earlier, communities obtained by modularity maximization may include vertices that can move from one group to another depending on the heuristic or the vertex ordering. The vertex groups obtained using constant communities are invariant under these algorithmic parameters and, thereby, provide a lower bound on the number of uniquely identifiable communities in the network. Although trivially each vertex can be considered to be a constant community by itself, our goal is to identify the largest number of vertices (i.e., at least three or more) that can be included in an invariant group.

The presence of such invariant structures can be used to evaluate the accuracy of the communities when other independent methods of verifications are unavailable. However in many networks, constant communities constitute only a small percentage of the total number of vertices. To understand how other non-constant vertices are allocated to communities, we show that by using constant communities we can significantly reduce the variations in results. Thus, building from the more accurate results reduces the variance over the larger network. In brief our main contributions are as follows:

- demonstrate the possibility of extreme variance in community structure due to vertex perturbations

- develop metrics to determine whether a network possess invariant groups of constant communities
- demonstrate how using constant communities as a pre-processing step can reduce the variance in modularity maximization methods.

\section{Results}

Experimental setup. In this section, we first demonstrate that even for the same optimization objective (in this case maximizing modularity) and the same heuristic, the inherent non-determinism of the method can significantly change the results. Based on our results, we define metrics to estimate the propensity of a network to form communities. Finally, we show how combining constant communities as a pre-processing step can help improve the modularity of the community detection algorithm for the network as a whole.

We selected two popular agglomerative modularity maximization techniques - the method proposed by Clauset et al. ${ }^{15}$ (henceforth referred to as the CNM method) and the method proposed by Blondel et al. ${ }^{16}$ (henceforth referred to as Louvain method). Both these methods initially start by assigning one vertex per community. Then at each iterative step, two communities whose combination most increases the value of modularity are joined. This process of joining community pairs is continued until the value of modularity no longer increases. The Louvain method generally produces a higher value of modularity than $\mathrm{CNM}$, because it allows vertices to migrate across communities if that leads to a more optimum value.

In order to identify these communities, for each network in the test suite, we applied the CNM (and Louvain) method over different permutations of the vertices and then isolated the common groups that were preserved across the different orderings (see Methods section). These common groups of vertices were marked as the constant communities for the respective network.

We identified constant communities using both the CNM and Louvain algorithms. We observed based on the high $(>0.80)$ Normalized Mutual Information (NMI) ${ }^{27}$ (see the supplementary information for the definition of NMI) values that the overlap between the constant communities obtained from the two methods is considerable ${ }^{28,29}$ (see Table III in the supplementary information). We have further detected the constant communities using another very popular non-deterministic community finding algorithm called 
Infomap ${ }^{33}$ which is not an agglomerative method but tries to minimize the minimum description length of the bit sequence generated by a random walk. We similarly observed the high overlap between the constant communities obtained from Louvain and Infomap (see Table III in the supplementary information). Therefore, in the interest of space and clarity we confine our discussion about the properties of constant communities to those obtained from the Louvain method.

Degree preserving order. Ideally, the total number of different orderings to be tested should be equal to the factorial of the number of vertices in the network. However, even for the smallest network in our set (Chesapeake with 39 vertices) this value is astronomical. We therefore restrict our permutations to maintain a degree-preserving order. The vertices are ordered such that if degree of $v_{i}$ is greater than the degree of $v_{j}$, then $v_{i}$ is processed prior to $v_{j}$.

In addition, to reducing the number of vertex permutation, degree-preserving permutation also has another important advantage. Recall that the networks in the test suite have few vertices with high degrees and a lot with low degrees. Therefore, arranging the high degree vertices earlier pushes most of the fluctuations towards the later part of the agglomeration process. This ensures that the subcommunities formed initially are relatively constant and only later do the divergence in community memberships take place. Clearly, such orderings based on decreasing degrees are geared towards facilitating low variance in communities. If even this ordering does not produce constant structures, it makes a very strong case about the inherent fluctuations that underlie modularity maximization methods.

Test suites. Our experiments were conducted on networks obtained from real-world data as well as on a set of synthetically generated networks using the LFR model ${ }^{17}$. The set of real-world networks is obtained from the instances available at the 10th DIMACS challenge website $^{18}$. The networks, which are undirected and unweighted, include - Jazz (network of jazz musicians; $|V|=198,|E|=$ $2742)^{19}$, Polbooks (network of books on USA politics; $|V|=105$, $|E|=441)^{20}$, Chesapeake (Chesapeake bay mesohaline network; $|V|=39,|E|=340)^{21}$, Dolphin (Dolphin social network; $|V|=62$, $|E|=159)^{22}$, Football (American college football; $|V|=115,|E|=$ $1226)^{23}$, Celegans (Metabolic network of C. elegans; $|V|=453,|E|=$ $2025)^{24}$, Power (topology of the Western States Power Grid of the USA; $|V|=4941,|E|=6594)^{25}$ and Email (e-mail interchanges between members of the Univeristy Rovira i Virgili; $|V|=1133$, $|E|=5451)^{26}$ (note that $|V|$ refers to the number of vertices and $|E|$ refers to the number of edges). All these networks exhibit scale-free degree distribution (see Figure S1 in the supplementary information).

Networks generated using the LFR model are associated with a mixing parameter $\mu$ that represents the ratio of the external connections of a node to its total degree. We created LFR networks based on the following parameters ${ }^{8}$ : number of nodes $=500$, average degree $=$ 20 , maximum degree $=50$, minimum community size $=10$, $\max$ imum community size $=50$, degree exponent for power law $=2$, community size exponent $=3$. We varied the value of $\mu$ from $0.05-$ 0.90 . Low values of $\mu$ correspond to well-separated communities that are easy to detect and consequently these networks contain larger percentage of constant communities. As $\mu$ increases, communities get more ambiguous and community detection algorithms provide more varied results leading to fewer vertices being in significantly sized constant communities.

Sensitivity of community structure to vertex perturbations. In our first experiment we study how the community structures of the networks change under vertex perturbations. Since constant communities are the groups of vertices that remain invariant, we measure the change in community structure based on the number of constant communities. We define sensitivity $(\phi)$ as the ratio of the number of constant communities to the total number of vertices. If $\phi$ is 1 then each vertex by itself is a constant community (the trivial case), thus there is no consensus at all over the set of communities obtained over different permutations. The higher the sensitivity metric, the fewer the vertices in each constant community and, therefore, this metric is useful for identifying networks that do not have a good community structure under modularity maximization.

The sensitivity of each network is given in Figure 2. The $\mathrm{x}$-axis indicates the number of different permutations of the vertices and the $y$-axis plots the value of the sensitivity. We observe that for most of the networks the number of constant communities become stable within the first 100 permutations, and the sensitivity values are low. This indicates that there can potentially exist very strong groups in these networks that have to be together to achieve high modularity. However, for networks such as Power grid and Email, the number of constant communities kept increasing until the values of $\phi$ were close to 1 . Thus, the community detection results for these two networks

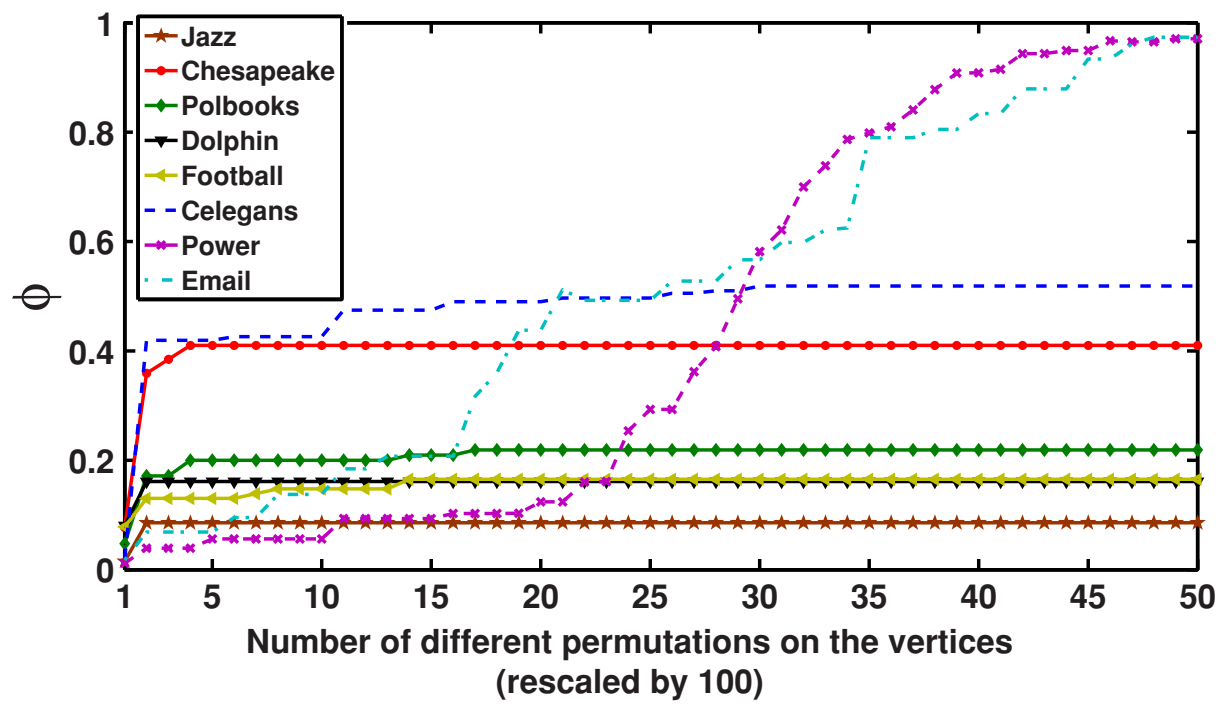

Figure $2 \mid$ Sensitivity of each network across 5000 permutations. X-axis indicates the number of permutations. The x-axis is rescaled by a constant factor of 100 for better visualization. Y-axis indicates the value of sensitivity as it changes over the permutations. Power and Email networks have very high sensitivity values indicating that they possibly do not have a tightly knit community structure. 


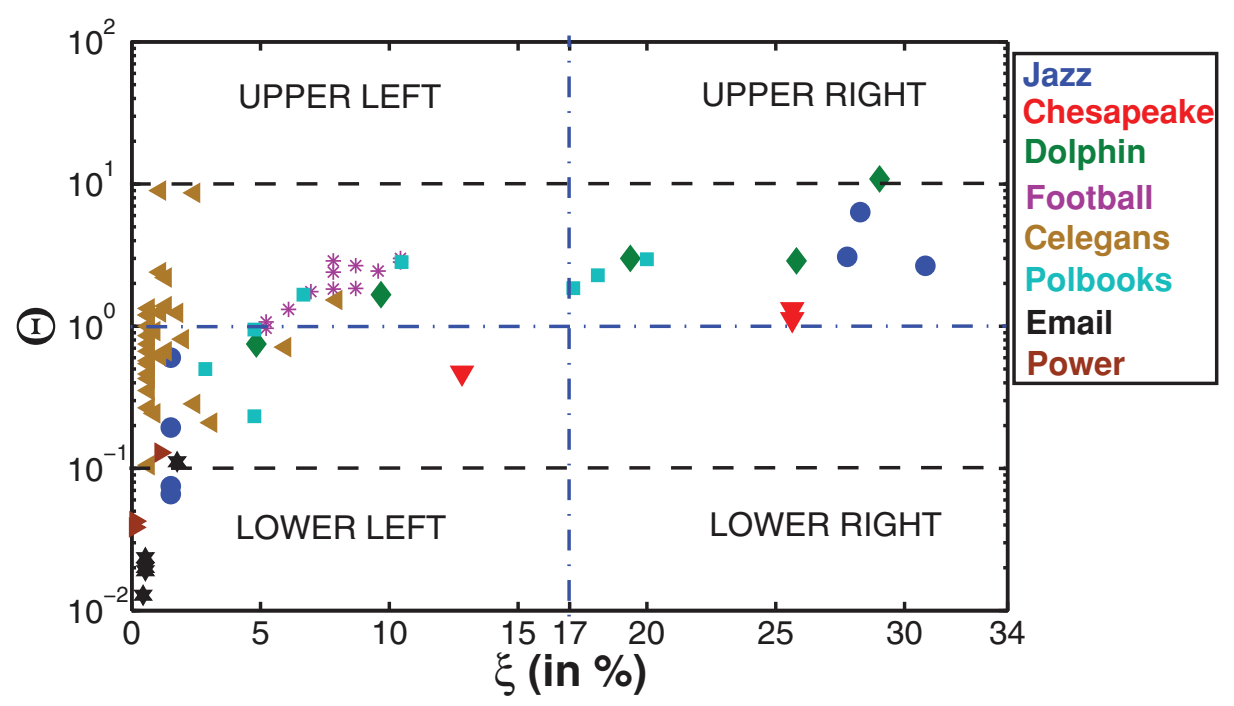

Figure 3 Comparison between the relative size and strength of the constant communities. X-axis plots the relative size in percentage. Y-axis (in logarithmic scale) plots the strength. Jazz, Dolphin, Polbooks and Chesapeake show strong constant community structure. But Email and Power hardly have any constant communities. The plot is vertically divided at $\mathrm{x}=17$ that could help systematically analyze the distribution of the points.

are extremely sensitive to the vertex perturbations. This implies that the communities (if any) in these two networks are not tightly knit i.e., very "amorphous".

Percentage of constant communities. We now investigate, in further detail, the properties of constant communities. We define the relative size $(\xi)$ of a constant community as the ratio of the number of vertices in that constant community to the total number of vertices in the network and the strength $(\Theta)$ as the ratio of the edges internal to the constant community to the edges external to the constant community.

Figure 3 plots the relative size (in percentage) of the constant communities with respect to their strength. If the strength of a constant community is above 1 (above 0 in log scale) then the number of internal edges in the community is larger than the number of external edges. The higher the value, the more tightly connected is the community. We see that the value of relative size ranges from $0-34$, with a larger cluster of values around $0-5$. This shows that most of the constant communities contain very few vertices with respect to the network size. If the relative size of the constant communities is low then the remaining vertices have more freedom in migrating across communities, making the community structure weaker. We observe that, despite there being more constant communities of low relative size, there are some networks that have multiple constant communities with relative size over $15 \%$ of the total number of nodes indicating that they have a much stronger community structure. These include Jazz, followed by Dolphin and then Polbooks and Chesapeake.

Relative size and strength together provide an estimate of which networks have good community structure. If we divide the $\mathrm{x}$-axis at roughly the mid-point of the range and the $y$-axis at 1 , then we obtain four quadrants each representing different types of community structures. The first quadrant (upper right) contains communities that have high relative size as well as high strength. Networks containing a large number of such constant communities are less likely to be affected by perturbations. Diagonally opposite is the third quadrant (lower left), which contains communities of low relative size and low strength. As discussed earlier, networks having communities predominantly from this quadrant will produce significantly different results under perturbations and are likely to not have a strong community structure under modularity maximization. The second quadrant (upper left) contains the groups of vertices that are strongly connected but have small relative size. This indicates that there are some pockets of the network with strong community structure. The fourth quadrant (lower right) represents communities with high relative size but low strength. In this set of experiments it is empty, and we believe that this area will be sparsely populated, if at all. This is because networks having such communities will have a very special structure: strongly connected groups of very few vertices with many spokes radiating out to account for the high number of external communities.

Pull from external connections. We note in Figure 3 that there are several constant communities whose strength is below one, i.e., they have more external than internal connections. This is counterintuitive to the idea that a strong community should have more internal connections. Indeed, modularity maximization methods always tend to create communities whose strengths are greater than one. However, the structure of some of the constant communities belies this convention.

We observe that in these cases, the external connections are distributed across different communities. Furthermore, the number of connections to any one external community is always lower than the internal connections. Based on this observation, we hypothesize that a group of vertices are likely to be placed together so long as the internal connection is greater than the connections to any one single external community. Then the vertices within the community do not experience a significant "pull" from any of the external communities that will cause them to migrate, and, therefore, their propensity to remain within their own communities is high. We quantify this measurement as follows:

Let $v$ be a vertex in a constant community; further, let $D(v)$ denote the degree of $v$, and $E N(v)$ and $I N(v)$ denote the number of external and internal neighbors of $v$ respectively (i.e., $D(v)=I N(v)+E N(v)$ ). We also assume that the $E N(v)$ external neighbors are divided into $k$ external groups, and $E N G(v)$ denote a set of $k$ elements where the $i$ th element in the set represents the number of neighbors of $v$ belonging to the $i$ th external group. For instance, consider the vertex $A$ in $C C_{1}$ in Figure 4 (Top), $D(A)=9, I N(A)=3, E N(A)=6$ and $E N G(A)=$ $\{3,2,1\}$ (i.e., three external neighbors in $C_{2}$, one external neighbor in $C C_{3}$, and two external neighbors in $C C_{4}$ ). Similarly, we calculate $\operatorname{ENG}(v)$ for each vertex in the network and form a list $\operatorname{DENG}(G)$ by taking union over all $\operatorname{ENG}(v)$, that is, only unique entries across $\operatorname{ENG}(v)$ get listed in $\operatorname{DENG}(G)$ (see Figure 4-top). The list is then ranked in ascending order i.e., the group with lowest number of 


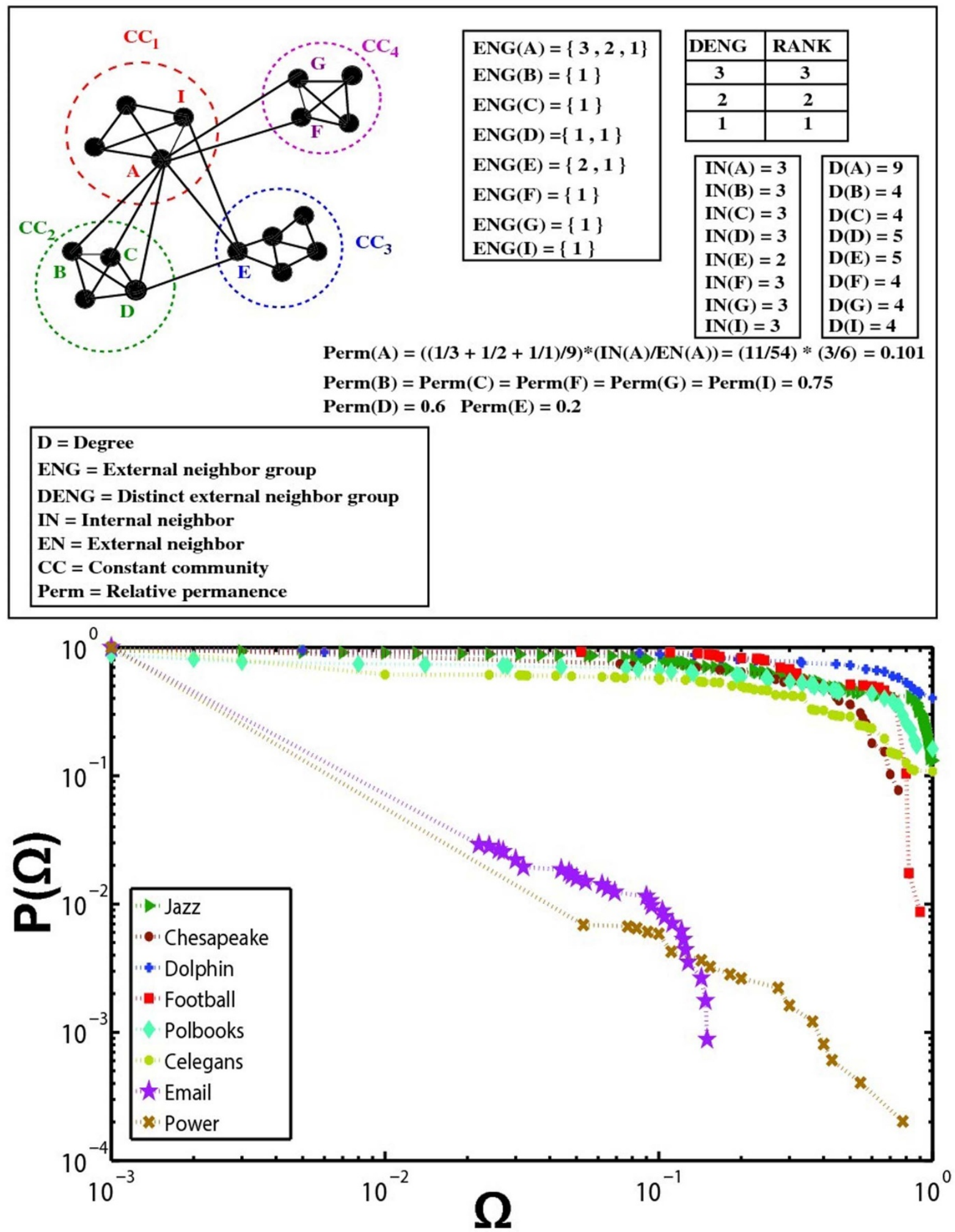

Figure 4 Top: Schematic diagram illustrating the computation of the relative permanence of the vertices. Bottom: Distribution of relative permanence values. X-axis plots the value of $\Omega$ and y-axis plots the cumulative fraction of vertices $(P(\Omega))$ exhibiting that $\Omega$. Both axes are in logarithmic scale.

external neighbors is ranked 1 , the group with second lowest external neighbors is ranked 2 and so on. The intuition behind this ranking is that we are more interested in how distinct the external neighbor groups are, rather than the absolute size of the external neighbor groups. Moreover, by ranking, we can reduce the skewness of the range of external group size. This rank would therefore signify the intensity of the pull of the particular external community and its inverse signifies the degree of stability of the vertex $v$. This formulation is motivated by the standard statistical measure called Mean Reciprocal Rank (MRR) ${ }^{32}$ which is the average of the reciprocal ranks of results for a sample of queries.

For a particular vertex, if the inverse rank of each of the external group is equal to one, it would point to the fact that all its external neighbors are diversely distributed (i.e., well-sparsed), and therefore 
the pull experienced should be minimum; in contrast, if the value is much lower than one, it would imply that the vertex experiences a strong pull from its external neighbors. We define the strength of a vertex $v, \theta(v)$, as the ratio of the internal neighbor $(I N(v))$ to the external neighbor $(E N(v))$ of vertex $v$ similar to the strength $(\Theta)$ of a constant community defined earlier. Mathematically, the suitably normalized value of relative permanence, $\Omega(v)$, of a vertex $v$ in a constant community can be expressed as:

$$
\Omega(v)=\theta(v) \times \frac{\sum_{i=1}^{k} \frac{1}{\operatorname{Rank}\left(E N G_{i}(v)\right)}}{D(v)}
$$

where $\operatorname{Rank}\left(\operatorname{ENG}_{i}(v)\right)$ denote the rank (retrieved from the DENG(v) list) of the $i$ th element in $E N G(v)$. This metric indicates the propensity of a vertex to remain in the same community regardless of any algorithmic parameters.

Figure 4 (Top) presents a schematic diagram for computing relative permanence of vertices within the communities. Figure 4 (Bottom) plots the cumulative distribution of the relative permanence over the vertices in all networks. The $\mathrm{x}$-axis indicates the value of the relative permanence and the y-axis, the cumulative fraction of vertices having the corresponding relative permanence value. The nature of the cumulative permanence distribution of the vertices is roughly same for all networks except Email and Power. The distinguishing nature of the curves for Email and Power graphs compared to the other graphs indicates that very few number of vertices in these two networks have higher relative permanence values and therefore experience more "pull" from the external communities. Another observation is that a high fraction of vertices in Jazz, Polbooks, Dolphin and Celegans have relative permanence close to one. These vertices are more "stable" compared to the other vertices in the respective networks.

Constant communities for improving the modularity. We note that in many networks (such as Football and Celegans) constant communities form only a small percentage of the vertices. Thus, finding only the constant communities may not provide adequate information about the relationship amongst the rest of the vertices.
We therefore leverage on the invariant results in the first and second quadrants of Figure 3 as building blocks to identify larger communities.

We first permute the vertices 5000 times in degree-descending order i.e., each of the permutations preserves the constraint that if vertex $v_{i}$ is placed before $v_{j}$ in the sequence then $\operatorname{degree}\left(\mathbf{v}_{\mathbf{i}}\right) \geq$ $\operatorname{degree}\left(\mathbf{v}_{\mathbf{j}}\right)$. Then for each of these permutations, we run Louvain algorithm and obtain the community structure (and the modularity value). Table I (left) shows the mean modularity (and its variance) obtained by averaging the modulatity values of all iterations. Next from these community structures obtained across the different permutations, we detect the constant communities (see the Methods section and Algorithm 1 in supplementary information) and combine them into super-vertices. This process creates a smaller network as well as ensures that the vertices in the constant communities always stay together. Then we execute a modularity maximization algorithm over the entire network (see Methods section). We compute the variance in results by executing the underlying modularity maximization algorithm individually over 5000 permutations, in each case maintaining the degree-preserving order. As shown in Table I (left), combining constant communities as a pre-processing step both increases the mean modularity value as well as reduces the variability across permutations for real-world networks.

We also observe that the variance becomes 0 or very low for the networks which have significant number of constant communities in the first and second quadrants of Figure 3. The results obtained from the other networks with high sensitivity, such as Email and Power, still indicate some variance although the value is less pronounced.

These observations on real-world networks lead us to believe that pre-processing using constant communities is more effective if a network has strong community structure. To test this hypothesis, we created LFR graphs with mixing parameters from 0.05 to 0.90 . Low mixing parameters indicate strong community structure. For the LFR graphs, we repeat the same set of experiments as discussed above and obtain the mean modularity and its variance. As shown in Table I (right), pre-processing using constant communities also helps increase the modularity value and reduces variability of the results in the LFR graphs.

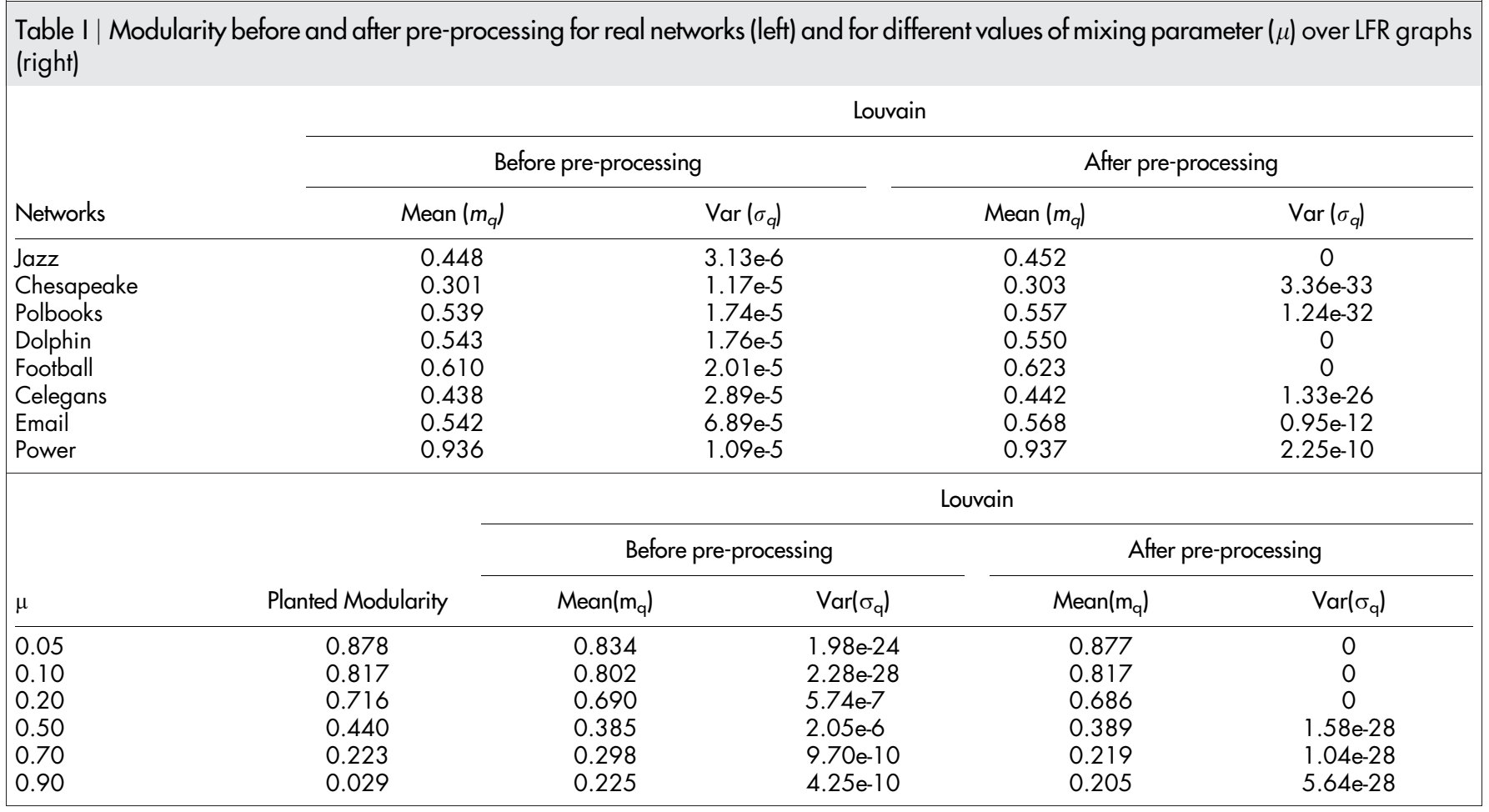




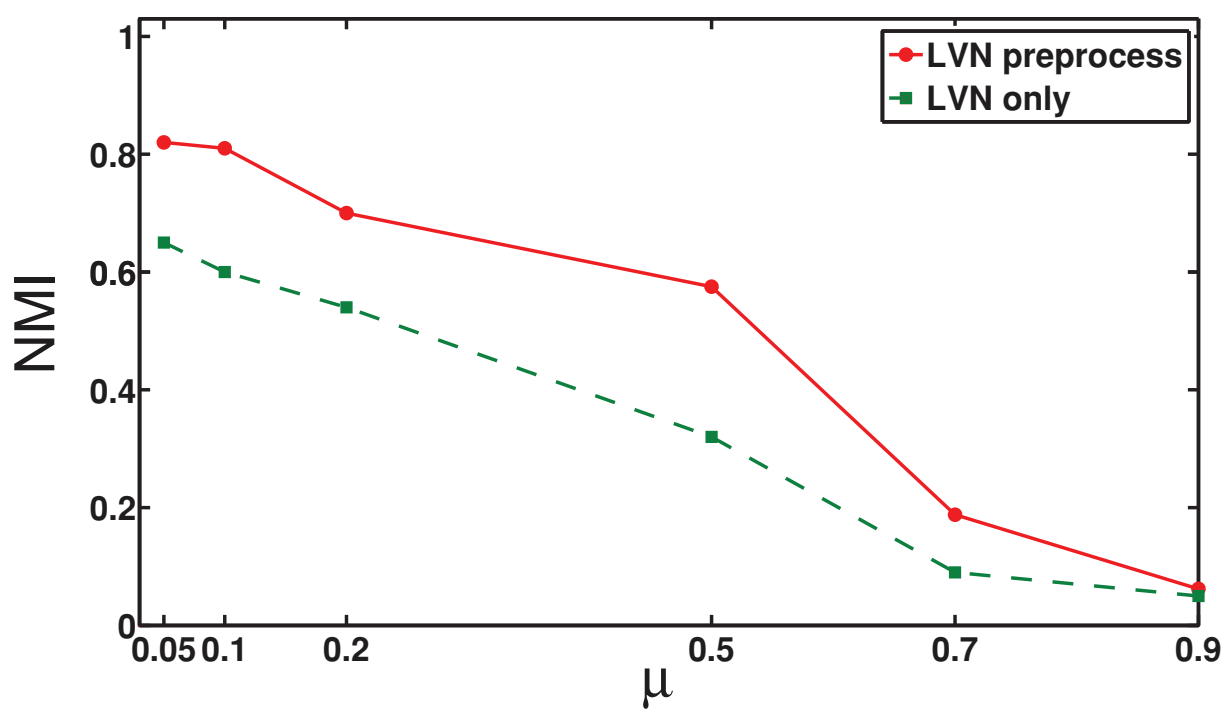

Figure 5 | Variation of NMI for different values of mixing parameters. The broken line corresponds to the experiment without the pre-processing step and the solid line to the experiment after using the pre-processing step.

Another advantage of LFR networks is that we know the "ground truth" i.e., the correct distribution of communities (exact number of vertices in each community and the number of in-community connections between them). We used NMI to compare the communities obtained, with and without using the pre-processing step with the ground truth community structures of LFR graphs for different mixing parameters. As shown in Figure 5, when the community structure is strong (low mixing parameter), using constant communities pushes the result towards the ground truth. In contrast, when the community structure is not well-defined (high mixing parameter), use of constant communities does not mimic the community distribution of the ground truth, because there can be many variations of community distribution in such networks that lead to high modularity. These results once again highlight the significance of constant communities.

Relative ranking of constant communities. A constant community is meaningful if it is large in size (high $\xi$ ) or it has high relative permanence $(\Omega)$. We calculated the relative permanence of a constant community by averaging the relative permanence of its constituent vertices. We experimented to see which one of these two properties is more important in determining high modularity. To do so, we ordered the constant communities according to (a) decreasing order of $\xi$ and (b) decreasing order of $\Omega$. We combined the constant communities into super-vertices one by one following the order obtained from (a) and (b) separately. After each combination, we computed the modularity and compared the value with the average modularity (over 5000 permutations) obtained by using the Louvain method without any pre-processing.

Figure 6 compares the modularity obtained by collapsing constant communities according to the order obtained from (a) (dotted blue line) and (b) (dotted green lines). For almost all the networks, there is a transition where the modularity values cross over the mean modularity (solid red line). Once this transition takes place, the modularity values generally remain above (or at least equal to) the mean modularity. This critical point indicates the smallest fraction of

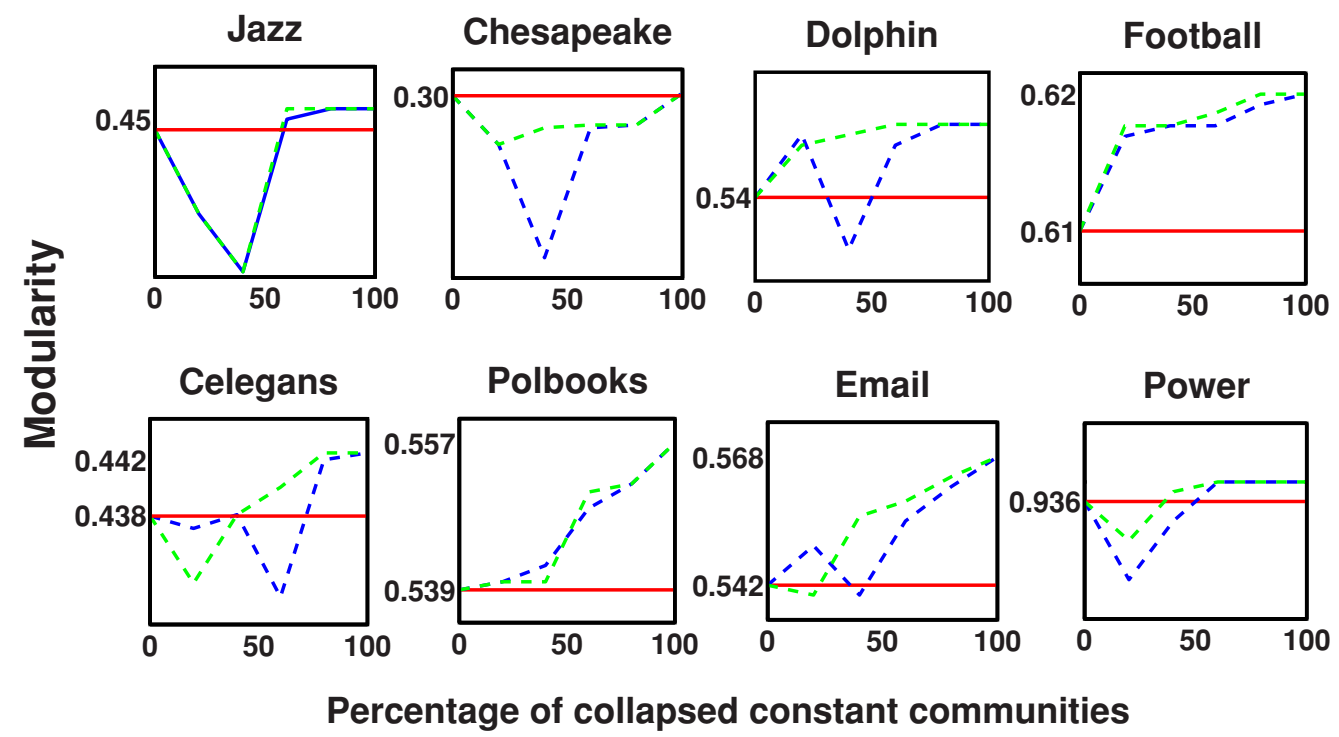

Figure 6 Modularity after partially collapsing the constant communities. The broken blue lines are in decreasing order of size and the broken green lines are in decreasing order of relative permanence. The red lines depict the mean modularities without using constant communities. 
Table II | Few constant communities of PhoNet and the features they have in common

Constant communities

$/ \mathrm{p}^{\mathrm{h}} /, / \mathrm{t}^{\mathrm{h}} /, / \mathrm{k}^{\mathrm{h}} /$

$/ \mathrm{mb} /, /{ }^{\mathrm{n}} \mathrm{d} /, /{ }^{\mathrm{n}} \mathrm{g} /$

$/ \mathrm{p} /, / \mathrm{t} /, / \mathrm{k} /$

$/ \mathrm{t} /, / \mathrm{d} /, / \mathrm{n} /$

$/ \mathrm{V}, / \mathrm{n} /, / \mathrm{t} /, / \mathrm{d} /$

constant communities required to outperform the original algorithms. We observe further that the green lines (ordered according to $\Omega(v)$ ) generally reach the critical point earlier than the blue lines (ordered according to $\xi$ ), indicating that $\Omega(v)$ is a better indicator of constant communities.

Case study. The significance of constant community in a network can be further understood if we consider networks where nodes have specific functionalities associated with them. We hypothesize that in such a network a constant community would represent indispensable functional blocks that reflect the defining characteristics of the network. In order to corroborate this hypothesis we conduct a case study on a specific type of linguistic network constructed from the speech sound inventories of the world's language ${ }^{30}$. The sound inventory of a language comprises a set of consonants and vowels also sometimes together known as phonemes. In order to unfurl the co-occurrence principles of consonant inventories, the authors ${ }^{30}$ constructed a network (phoneme-phoneme network or PhoNet) where each node is a consonant and an edge between two nodes denotes if the corresponding consonants have co-occurred in a language. The number of languages in which the two nodes (read consonants) co-occur defines the weight of the edge between these nodes. Note that each node here has a functional representation since it can be represented by means of a set of phonetic features (e.g., bilabial, dental, nasal, plosive etc) that indicate how it is articulated. Since this is a weighted graph, we suitably define a threshold to construct the unweighted version. We compute constant communities of PhoNet and observe that each such graph (see Table II) represents a natural class, i.e., a set of consonants that have a large overlap of the features ${ }^{30}$. Such groups are frequently found to appear together across languages, and linguists describe this observation through the principle of feature economy ${ }^{30}$. According to this principle, the speakers of a language tend to be economic in choosing the features in order to reduce their learning effort. For instance, if they have learnt to use a set of features by virtue of learning a set of sounds, they would tend to admit those other sounds in their language that are combinatorial variations of the features already learnt - if a language has the phonemes $/ \mathrm{p} /$ (voiceless, bilabial, plosive), /b/ (voiced, bilabial, plosive) and /t/ (voiceless, dental, plosive) in its inventory then the chances that it will have /d/ (voiced, dental, plosive) is disproportionately higher compared to any other arbitrary phoneme since by virtue of learning to articulate $/ \mathrm{p} /, \mathrm{b} /$ and $/ \mathrm{t} /$ the speakers need to learn no new feature to articulate /d/. Identification of constant communities therefore systematically unfolds the natural classes and provides a formal definition for the same (otherwise absent in the literature). We plot in Figure S2 (see supplementary information), the average hamming distance between the feature vectors of phonemes forming a constant community versus the community size. The average hamming distance is significantly lower in the case when a set of randomly chosen phonemes are grouped together and assumed to represent a community with varying sizes as that of the constant communities. Further, we observe that collapsing the constant communities results either in more dilute groups (still with a certain degree of feature overlap) or reproduces the same constant communities indicating that no valid dilution is possible for these functional blocks.

\section{Discussion}

Constant communities are regions of the network whose community structure is invariant under different perturbations and community detection algorithms. They, thereby, represent the core similar relationships in the network. The existence of multiple results for community detection is well known; however, this is one of the first studies of the invariant subgraphs that occur in a network.

Although we currently detect constant communities by comparing across different permutations, our results have uncovered some interesting facets about the community structure of networks, which can lead to improved algorithms for community detection. First, we observe that constant communities do not always have more internal connections than external connections. Rather, the strength of the community is determined by the number of different external communities to which it is connected. We have proposed a metric to quantify the pull that a vertex experiences from the external communities and the relative permanence of the said vertex indicating its inertia to stay in its own community.

Secondly, in most networks, constant communities cover only a subset of the vertices. Depending on the size of the constant communities it may not be correct or necessary to assign every vertex to a community, as is the focus of most community detection algorithms. Furthermore, even if when we insist on assigning a community to each vertex, the constant communities can be leveraged to produce results with higher modularity and lower variance. Thus, as discussed earlier, constant communities form the smallest indivisible units in the networks and particularly in the case of agglomerative methods can be used to hierarchically build larger communities.

Thirdly, the high functional cohesion among the vertices of the constant community can render meaning to the community structure of the networks. This conclusion is much more apparent for labeled graphs where the vertices are associated with certain functional properties. If we stop at detecting only the constant communities and treat them as the actual community structure of the graph, we observe that sometimes it acts as a hard bound since no further community detection might be possible. Therefore, we suggest that the prior detection of these building blocks is always significant in order to further decide to merge them into more coarse-grained communities pertaining to a diluted functional cohesion.

The fourth and most important observation is that not all networks have significant constant community structure. The two most egregious examples in our test suites are Power and Email graphs. The absence of constant communities in the networks indicate that either communities in general do not exist or they are highly overlapped and therefore do not have a significant constant region. The first case is true for Power grid, which as a grid is unlikely to have communities. We believe that the second reason probably holds for the Email network. A set of professional emails within correspondents in the same university is likely to have more overlaps than clear cut communities.

Finally, we have demonstrated evidence that the modularity measure is not enough to judge the inherent compartmental structure of a network. For instance, Email and Power networks have reasonably higher modularities compared to the others. Still, no consensus is observed in their community structures. Rather their sensitivity measures indicate that each node might act as individual constant community in the further iterations. Therefore, the goodness metric of the community detection algorithm should be redefined in a way that can effectively capture the modular structure of the network.

We note that the experiments in this paper focused solely on agglomerative modularity maximization methods. We plan to continue our studies on the effect of vertex perturbations on other types of community detection algorithms such as divisive and spectral 
methods as well as different optimization objectives. In particular we are very keen to understand how the randomness of a network could be quantified in order to develop algorithms that take into account the variation in randomness of connections for determining the quality of the communities.

\section{Methods}

Identifying constant communities. In order to identify constant communities we permute the order of the vertices, and then apply a community detection algorithm to each of the permuted networks. The results vary across permutations. We select the groups of vertices that were always allocated together across all the permutations and mark them as constant communities. Algorithm 1 in supplementary information formalizes the steps to find out constant communities (see Figure S3 for the schematic diagram of the algorithmic steps in the supplementary information). The rationale behind this process is that these vertices must have some intrinsic connectivity properties that force them to stay together under all orderings.

To implement the vertex permutation, we adopt a stochastic degree-preserving scheme that can arrange the vertices based on the descending order of their degrees. The ordering of the set of vertices with the same degree is permuted. By applying this method we preserve the relative ordering of the degrees of the vertices since it is wellknown that node-degrees constitute a fundamental network property. We have also observed that the random permutations producing high modularity usually preserve a degree-descending order of vertices and the ones that result in low modularity usually are outcomes of cases where the algorithm would start executing from a lowdegree vertex. Thus, our permutations prevent us from the possibility of getting confined in a local maximum of the modularity.

Combining constant communities for modularity maximization. For these tests, we first collapse the constant communities to individual nodes (see Figure S3 in the supplementary information). This step ensures that the constant vertices are always grouped together and are guaranteed to remain within the same community. The total number of edges between the vertices of the two collapsed communities is computed and this sum is assigned as the new edge weight between them. We then apply a community detection method to the new weighted network to obtain the final modularity.

1. Lancichinetti, A. \& Fortunato, S. Community detection algorithms: a comparative analysis. Phys. Rev. E. 80, 056117 (2009).

2. Porter, M. A., Onnela, J. P. \& Mucha, P. J. Communities in networks. Notices of the American Mathematical Society. 56, 1082-1097 \& 1164-1166 (2009).

3. Newman, M. E. J. \& Girvan, M. Finding and evaluating community structure in networks. Phys Rev. E. 69, 026113 (2002).

4. Brandes, U., Gaertler, M. \& Wagner, D. Experiments on graph clustering algorithms. 11th Europ. Symp. Algorithms. 2832, 568-579 (2003).

5. Good, B. H., Montjoye, Y. A. \& Clauset, A. The performance of modularity maximization in practical contexts. Phys. Rev. E. 81, 046106 (2010).

6. Leskovec, J., Lang, K. L., Dasgupta, A. \& Mahoney, M. W. Community structure in large networks: natural cluster sizes and the absence of large well-defined clusters. CoRR. abs/0810.1355 (2008).

7. Seifi, M., Guillaume, J. L., Junier, I., Rouquier, J. B. \& Iskrov, S. Stable community cores in complex networks. CompleNet, 87-98 (2012).

8. Lancichinetti, A. \& Fortunato, S. Consensus clustering in complex networks. Nature Scientific Reports. 2 (2012).

9. Delvenne, J. C., Yaliraki, S. N. \& Barahona, M. Stability of graph communities across time scales. Proc. Natl. Acad. Sci. USA. 107, 12755-12760 (2010).

10. Lai, D., Lu, H. \& Nardini, C. Enhanced modularity-based community detection by random walk network preprocessing. Phys. Rev. E. 81, 066118 (2010).

11. Ovelgonne, M. \& Geyer-Schulz, A. An ensemble learning strategy for graph clustering. 10th DIMACS Implementation Challenge Graph Partitioning and Graph Clustering (2012).

12. Gfeller, D., Chappelier, J. C. \& De Los Rios, P. Finding instabilities in the community structure of complex networks. Phys. Rev. E. 72, 056135 (2005).

13. Reidy, J., Bader, D. A., Jiang, K., Pande, P. \& Sharma, R. Detecting communities from given seeds in social networks. Technical Report, http://hdl.handle.net/1853/ 36980 (19.06.2012).

14. Srinivasan, S., Chakraborty, T. \& Bhowmick, S. Identifying base clusters and their application to maximizing modularity. Contemporary Mathematics. Graph partitioning and Graph Clustering. (Bader, D. A., Meyerhenke, H., Sanders, P. and Wagner, D. eds.), AMS-DIMACS, 141-156 (2012).
15. Clauset, A., Newman, M. E. J. \& Moore, C. Finding community structure in very large networks. Phys. Rev. E. 70, 066111 (2004).

16. Blondel, V. D., Guillaume, J. L., Lambiotte, R. \& Lefebvre, E. Fast unfolding of community hierarchies in large networks. J. Stat. Mech. 2008 (10), P100081 (2008).

17. Lancichinetti, A. \& Fortunato, S. Benchmarks for testing community detection algorithms on directed and weighted graphs with overlapping communities. Phys. Rev. E. 80, 016118 (2009).

18. 10th DIMACS Implementation Challenge - Graph Partitioning and Graph Clustering. http://www.cc.gatech.edu/dimacs10/archive/clustering.shtml (12.01.2012).

19. Gleiser, P. \& Danon, L. Jazz musicians network: List of edges of the network of Jazz musicians. Adv. Complex Syst. 6, 565 (2003).

20. Kreb, V. Books on US Politics. http://www.orgnet.com (18.04.2012).

21. Baird, D. \& Ulanowicz, R. E. The seasonal dynamics of the Chesapeake Bay ecosystem. Ecol. Monogr. 59, 329-364 (1989).

22. Lusseau, D. et al. The bottlenose dolphin community of doubtful sound features a large proportion of long-lasting associations. Behavioral Ecology and Sociobiology. 54, 396-405 (2003)

23. Girvan, M. \& Newman, M. E. J. Community structure in social and biological networks. Proc. Natl. Acad. Sci. USA. 99, 7821-7826 (2002).

24. Duch, J. \& Arenas, A. Community identification using extremal optimization. Phys. Rev. E. 72 (2005).

25. Watts, D. J. \& Strogatz, S. H. Collective dynamics of 'small-world' networks. Nature. 393, 440-442 (1998).

26. Guimera, R., Danon, L., Diaz-Guilera, A., Giralt, F. \& Arenas, A. Self-similar community structure in a network of human interactions. Phys. Rev. E. 68, 065103(R) (2003).

27. Manning, C. D., Raghavan, P. \& Schutze, H. Introduction to Information Retrieval. Cambridge University Press. 1st Edition (2008).

28. Vinh, N., Epps, J. \& Bailey, J. Information theoretic measures for clusterings comparison: is a correction for chance necessary? Proceedings of the 26th Annual International Conference on Machine Learning. 1073-1080 (2009).

29. Strehl, A. \& Ghosh, J. Cluster ensembles - a knowledge reuse framework for combining multiple partitions. J. Mach. Learn. Res. 3, 1532-4435 (2003).

30. Mukherjee, A., Choudhury, M., Basu, A. \& Ganguly, N. Modeling the cooccurrence principles of the consonant inventories: a complex network approach. International Journal of Modern Physics C. World Scientific Publishing Company. 18, 281-295 (2008).

31. Delmotte, A., Tate, E. W., Yaliraki, S. N. \& Barahona, M. Protein multi-scale organization through graph partitioning and robustness analysis: application to the myosin-myosin light chain interaction. Phys Biol. 8, 055010 (2011).

32. Radev, D. R., Qi, H., Wu, H. \& Fan, W. Evaluating Web-based Question Answering Systems. LREC (2002).

33. Rosvall, M. \& Bergstrom, T. Maps of information flow reveal community structure in complex networks. Proc. Natl. Acad. Sci. USA. 105, 1118 (2008).

\section{Acknowledgements}

This work has been supported by the Google India PhD fellowship Grant in Social Computing, ITS project grant through Samsung GRO programme, College of IS\&T at University of Nebraksa at Omaha (UNO) and the FIRE and GRACA grants from the Office of Research and Creative Activity at UNO.

\section{Author contributions}

T.C., S.B., N.G., A.M. designed research; T.C., S.S., S.B., N.G., A.M. performed research; T.C., S.S., S.B., N.G., A.M. contributed new reagents/analytic tools; T.C., S.S., S.B., N.G., A.M. analyzed data and T.C., S.B., N.G., A.M. wrote the paper.

\section{Additional information}

Supplementary information accompanies this paper at http://www.nature.com/ scientificreports

Competing financial interests: The authors declare no competing financial interests.

License: This work is licensed under a Creative Commons

Attribution-NonCommercial-NoDerivs 3.0 Unported License. To view a copy of this license, visit http://creativecommons.org/licenses/by-nc-nd/3.0/

How to cite this article: Chakraborty, T., Srinivasan, S., Ganguly, N., Bhowmick, S. \& Mukherjee, A. Constant Communities in Complex Networks. Sci. Rep. 3, 1825; DOI:10.1038/srep01825 (2013) 